\title{
STUDY OF RISK FACTORS FOR NEONATAL MORTALITY DUE TO BIRTH ASPHYXIA IN A RURAL MEDICAL CENTRE, MAHARASHTRA
}

\author{
Mridula Srivastava1 ${ }^{1}$, Abhishek Srivastava², Ajay Pratap ${ }^{3}$ \\ ${ }^{1}$ Assistant Professor, Department of Paediatrics, Mayo Institute of Medical Sciences, Barabanki, Uttar Pradesh. \\ ${ }^{2}$ Associate Professor, Department of Pulmonary Medicine, Mayo Institute of Medical Sciences, Barabanki, Uttar Pradesh. \\ ${ }^{3}$ Professor, Department of Paediatrics, Mayo Institute of Medical Sciences, Barabanki, Uttar Pradesh.
}

\begin{tabular}{l}
\hline ABSTRACT \\
\hline OBJECTIVE \\
To determine the maternal and fetal risk factors for perinatal asphyxia and to know the mortality in the asphyxiated babies. \\
METHODS \\
All the consecutive hospital live births were evaluated during the study period of one year. Out of this birth cohort, asphyxiated \\
babies were grouped into cases and non-asphyxiated group as controls. Maternal, intrapartum and neonatal variables were recorded \\
in all births. Data was analysed and various statistical tests of significance applied to find the risk of association of various factors to \\
birth asphyxia.
\end{tabular}

\section{RESULTS}

Amongst 4714 live births, there were 171 cases of birth asphyxia, providing the incidence of 36 per 1000 live births. Risk factors significantly associated and having most strong association with perinatal asphyxia was observed in Eclampsia (OR 25.5) followed in decreasing frequency by Prematurity (OR 21.3), Meconium staining of amniotic fluid (OR 9.21), Antepartum hemorrhage (OR 8), Gestational diabetes mellitus (OR 4), Breech presentation (OR 3.8), Oligohydramnios (OR 2.8), PROM (OR 2.6), PIH (OR 2.1). Asphyxia related mortality was 10.6 per 1000 live births.

\section{CONCLUSIONS}

There is a need to strengthen intrapartum management and early identification of mothers with high risk pregnancy to reduce asphyxia mortality and morbidity.

\section{KEYWORDS}

Birth Asphyxia, Risk Factors (Maternal and Fetal).

HOW TO CITE THIS ARTICLE: Srivastava M, Srivastava A, Pratap A. Study of risk factors for neonatal mortality due to birth asphyxia in a rural medical centre, Maharashtra. J Evolution Med Dent Sci 2016;5(2):127-130, DOI: 10.14260/jemds/2016/31

\section{INTRODUCTION}

The initial few minutes after birth are full of anxious moments and rapid physiological adjustments. Most of the babies (90\%) make the transition from intrauterine to extrauterine life without difficulty, $10 \%$ however may need varying degree of assistance to begin breathing at birth.

Interruption in this sequence of transition leads to oxygen deprivation leading further to depressed baby.(1) Birth asphyxia or Perinatal asphyxia refers to an impairment of the normal exchange of respiratory gases during parturition, and the ensuing adverse effects on the fetus. It is an important cause of fresh stillbirth and early neonatal death.(2)

The National Neonatology Forum of India has proposed that "Gasping and ineffective breathing or lack of breathing at 1-minute" should be designated as birth asphyxia.(3) which is consistent with the definition given by WHO as the "Failure to initiate and sustain breathing at birth." Birth asphyxia is an important cause of neonatal morbidity and mortality.

Financial or Other, Competing Interest: None.

Submission 18-12-2015, Peer Review 19-12-2015,

Acceptance 02-01-2016, Published 06-01-2016.

Corresponding Author:

Dr. Mridula Srivastava,

Assistant Professor,

Department of Paediatrics,

Mayo Institute of Medical Sciences,

Barabanki, Uttar Pradesh.

E-mail: mridula.srivastav@gmail.com

DOI:10.14260/jemds/2016/31
It has varying effects on the neonatal brain depending upon the gestational age of the baby and the severity and time of onset of the asphyxiating event(s), which can occur at any point in the infant's antepartum, intrapartum and postpartum life. Although, Hypoxic-Ischemic Encephalopathy (HIE) is the hallmark of severe asphyxia, such cases can often exhibit multisystem failure.

Of the 130 million new-born infants born each year globally, about 4 million die in the first 4 weeks of life- the neonatal period.(4) Most of the neonatal deaths (99\%) arise in low- and middle income countries and over half occur at home, where the bulk of deliveries take place.

It is estimated that around $23 \%$ of all new-born deaths are caused by birth asphyxia with a large proportion of stillbirths. Following improvements in primary and obstetric care in most industrialized countries, the incidence of birth asphyxia has reduced significantly to less than $0.1 \%$, whereas it is several folds higher in developing countries. (5)

Accurate estimates of the proportion of neonatal mortality attributable to birth asphyxia are limited by absence of vital registration in communities where the majority of neonatal deaths occur. Hospital-based studies may substantially underestimate the burden in rural areas, where early deaths most of which occur at home (About 83\% in rural India), are more likely to be under-reported.(5)

In rural regions of the Maharashtra and Uttar Pradesh, states of India, $25 \%$ and $23 \%$ of neonatal mortality were attributed to birth asphyxia, respectively. $(5,6)$ 


\section{MATERIAL AND METHODS}

This study was conducted in a neonatal care unit attached to Department of Pediatrics of Mahatma Gandhi Institute of Medical Sciences, Sevagram, a Rural Medical College, conducting approximately 4500 deliveries annually over a period of one year beginning from $1^{\text {st }}$ March 2010 to $28^{\text {th }}$ February 2011.

This was a hospital based retrospective and analytical study, which involved a population of all the live intramural deliveries conducted during the study period. The proportion of the asphyxiated neonates was determined. Various maternal and fetal risk factors associated with perinatal asphyxia were evaluated by a case control study. The population of babies who were born with birth asphyxia constituted the cases.

All the babies who had uneventful birth (Without birth asphyxia) during the study period were taken as controls. The proportions of various risk factors in the two groups were analysed to determine the strength of their association with birth asphyxia.

\section{Following Neonates were excluded from the Present Study}

- $\quad$ All the out born babies.

- Still born babies (Based on abdominal USG, Fetal Doppler and Clinical Parameters).

All the inborn hospital deliveries conducted during this study period were enrolled in the study. Each delivery was attended by a pediatric resident and babies needing resuscitation were resuscitated as per the National Resuscitation Programmes (NRP) Guidelines by IAP and AAP 2010.(1)

Detailed maternal profiles of all the babies were recorded on a pre-designed proforma with special emphasis on any complication of pregnancy like PIH, eclampsia, any chronic illness, GDM, ante-partum haemorrhage, oligohydramnios. Intrapartum factors like Premature Rupture Of Membrane (PROM), meconium staining of amniotic fluid, mode of delivery, instrumentation and the presentation of the baby were recorded.

The accurate birth weights taken on the electronic weighing machine in the NICU (Nearest to1gram), Sex, Apgar scores and gestational age of all the babies were recorded. Gestation was assessed by the date of Last Menstrual Period (LMP), where known. New Ballard scoring was used when LMP was not known.

The asphyxiated babies born with a low Apgar score were resuscitated and kept under observation in NICU. These neonates were appropriately managed following the standard protocols. These babies were further followed up until discharged from NICU and final outcome was recorded.
All the statistical data was assessed using Chi Square test and Chi square for trend test of statistical significance wherever applicable. The analysis was done using EPI info software and SPSS.

\section{RESULTS}

During the study period of one year, a total of 4714 neonates, who fulfilled the inclusion criteria, made up the study group. Out of these live born babies, 171 babies suffered from birth asphyxia (3.63\%). The incidence of birth asphyxia in our study was found to be 36 per 1000 live births. Out of total study cohort ( $n=4714), 53 \%$ were male babies and the females were $47 \%$.

\section{Risk Factor Analysis of Birth Asphyxia}

Various maternal and fetal factors known to be associated with a significant risk in causing birth asphyxia were observed and then analysed in this case control study.

\begin{tabular}{|c|c|c|c|c|c|}
\hline \multicolumn{2}{|c|}{ Risk Factor } & $\begin{array}{l}\text { Cases } \\
(171)\end{array}$ & $\begin{array}{l}\text { Control } \\
(4543)\end{array}$ & $\begin{array}{l}\mathbf{0} . \\
\mathbf{R} .\end{array}$ & $\begin{array}{c}\text { P. } \\
\text { Value }\end{array}$ \\
\hline \multicolumn{6}{|c|}{ Maternal Factors } \\
\hline 1. & $\mathrm{PIH}$ & $21(12.28)$ & $280(6.16)$ & 2.13 & $<0.05^{*}$ \\
\hline 2. & $\begin{array}{c}\text { Eclampsi } \\
\mathrm{a}\end{array}$ & $10(5.8)$ & $011(0.2)$ & 25.5 & $\begin{array}{c}<0.001 \\
* *\end{array}$ \\
\hline 3. & APH & $9(5.2)$ & 031(0.7) & 8.09 & $\begin{array}{c}<0.001 \\
* *\end{array}$ \\
\hline 4. & MSL & $50(29.2)$ & $195(4.3)$ & 9.21 & $\begin{array}{c}<0.001 \\
* *\end{array}$ \\
\hline 5. & $\begin{array}{c}\text { Oligohydr } \\
\text { amnios }\end{array}$ & $23(13.4)$ & $232(5.1)$ & 2.89 & $\begin{array}{c}<0.001 \\
* *\end{array}$ \\
\hline 6. & PROM & $16(9.35)$ & 168(3.69) & 2.69 & $\begin{array}{c}<0.001 \\
* *\end{array}$ \\
\hline 7. & GDM & $04(2.3)$ & $026(0.5)$ & 4.16 & $<0.05^{*}$ \\
\hline \multicolumn{6}{|c|}{ Fetal Factors } \\
\hline 1. & Pre Term & $61(35.6)$ & $114(2.5)$ & $\begin{array}{c}21.3 \\
8\end{array}$ & $\begin{array}{c}<0.001 \\
* *\end{array}$ \\
\hline 2. & Breech & $15(8.7)$ & $110(2.42)$ & 3.88 & $\begin{array}{c}<0.001 \\
* *\end{array}$ \\
\hline \multicolumn{6}{|c|}{$\begin{array}{l}\text { Table 1: Relationship of various } \\
\text { factors with birth asphyxia }\end{array}$} \\
\hline
\end{tabular}

Figures in parenthesis denotes percentage

${ }^{*} \mathrm{p}$ value is significant $(<0.05)$

${ }^{* *} p$ value is highly significant $(<0.001)$

Table 2 shows that of the total live births, 4539 (96.2\%) neonates were full term and $175(3.71 \%)$ were preterm. Out of the asphyxiated group, $103(2.29 \%)$ were full term neonates while 61 (34.8\%) neonates were preterm. This finding of higher proportion of asphyxia in preterms is significant with a significant $p$ value of $<0.05$ and Chi square of 532 .

\begin{tabular}{|c|c|c|c|c|c|c|}
\hline Group & $\begin{array}{c}\text { Gestational } \\
\text { Age }\end{array}$ & $\begin{array}{c}\text { Total No. } \\
(\mathbf{n = 4 7 1 4})\end{array}$ & $\begin{array}{c}\text { Birth Asphyxia } \\
(\mathbf{n = 1 7 1})\end{array}$ & \% & Chi-square & P value \\
\hline I & Term & 4539 & 103 & 2.29 & \multirow{2}{*}{532} & \multirow{2}{*}{$<0.05^{*}$} \\
\hline II & Pre term & 175 & 61 & 34.8 & \\
\hline \multicolumn{7}{|c|}{ Table 2: Relationship of Birth Asphyxia with } \\
Gestational Age in Total Live Babies \\
\hline
\end{tabular}

*Significant $\mathrm{p}$ value of $<0$. 


\begin{tabular}{|c|c|c|c|}
\hline & $\begin{array}{c}\text { No. of Asphyxiated } \\
\text { Babies } \\
(\mathbf{n = 1 7 1 )}\end{array}$ & $\begin{array}{c}\text { Mortality } \\
(\mathbf{n = 5 0 )}\end{array}$ & $\begin{array}{c}\text { P } \\
\text { Value }\end{array}$ \\
\cline { 1 - 3 } $\begin{array}{c}\text { Pre } \\
\text { Term }\end{array}$ & 61 & $\begin{array}{c}34 \\
(55.74 \%)\end{array}$ & $<0.05$ \\
\cline { 1 - 3 } Full & 103 & $\begin{array}{c}16 \\
(15.53 \%)\end{array}$ & \\
\cline { 1 - 2 } Term & \multicolumn{3}{|c|}{ Table 3: Mortality of cases in accordance } \\
with their gestational age \\
\hline
\end{tabular}

*p value is significant $(<0.05)$

Table 3 shows the asphyxia deaths amongst the case group. Out of the total 171 asphyxiated neonates, 61 babies were preterm of which 34 (55.74\%) expired due to asphyxia. 103 neonates were full term out of which 16 (15.53\%) babies died due to asphyxia. This higher proportion of deaths among the preterm neonates was analysed by applying chi square and $p$ value was statistically significant.

Hence the overall birth asphyxia related mortality was found to be 10.6 per 1000 live births and considering only the full term neonates, the asphyxia mortality comes out to be 3.3 per 1000 live births.

\section{DISCUSSION}

The clinical diagnosis of birth asphyxia along with the closely related conditions of hypoxic ischemic encephalopathy is recognised as an important cause of poor neurodevelopment outcome in a neonate. Of the estimated 130 million infants born each year worldwide, 4 million die in the first 28 days of life, the neonatal period.

In India approximately 9,40,000 neonatal deaths occurred during 2007, amongst which birth asphyxia contributed to about $19.5 \%$ in the mortality.(7) The prevalence of birth asphyxia in any community is to a large extent dependent on prevailing risk factors.

These factors in turn are influenced by the extent and impact of health education, literacy levels, socio-economic status, cultural and traditional beliefs affecting efficient utilisation of health care services as well as the quality of antenatal, obstetric and neonatal care. Understanding neonatal mortality in relation to these factors is crucial as it helps in reducing the number of deaths.

In our study, a total of 4714 live babies were born out of which 171 babies had asphyxia. The incidence of asphyxia in our study was found to be 36 per 1000 live births. This is comparable to a similar study by Chandra et al. ${ }^{(8)}$ who reported an incidence of 36.3 per 1000 live births. PIH was seen in $12.2 \%$ cases of birth asphyxia in the present study with an Odds Ratio of 2.13.

Eclampsia was found in $5.8 \%$ of the cases versus $0.2 \%$ in the control group with an Odds Ratio of 25.5 showing a strong and significant risk for asphyxia. Similar results were reported by S. Chandra et al.(8), Daga AS et al.(9) and Kaye D.(10) in their study also with PIH as a significant risk for birth asphyxia. In our study the proportion of APH was $5.2 \%$ in the asphyxiated group, whereas only $0.7 \%$ in the control group.

Asphyxia was present in $29.2 \%$ neonates born through MSL, while the control group had MSL in $4.3 \%$ babies. Sasikala et al.(11) and Nayak and Dalal.(12) reported asphyxia in $60.6 \%$ and $29.4 \%$ cases of MSL, respectively. PROM was found in $9.35 \%$ of cases of birth asphyxia as compared to $3.6 \%$ in controls. G Oswyn et al.(13) reported PROM as a factor associated with perinatal asphyxia with an OR of 3.96. Similar results were reported by Thangwijitra et al.(14) Anne CC et al.(6) Kaye D.(10)

Amongst fetal factors, premature labour is an independent risk factor for birth asphyxia. In the present study also, prematurity was associated as a risk factor in $35.6 \%$ of neonates amongst the asphyxiated group versus $2.5 \%$ in the control group. This view was supported by Anne CC et al.(6) A study at Prince Ali Ben Al Hussein Hospital, Jordan.(15) found a statistically significant association of gestational age as a risk factor for birth asphyxia, the incidence being more amongst preterms about $26.1 \%$ as compared with $11.8 \%$ amongst terms.

Out of the 171 asphyxiated neonates, mortality was seen in 50, hence asphyxia related mortality in this study was 10.6 per 1000 live births and considering the mortality only in full term babies this worked out to be 3.3 per 1000 live births.

\section{CONCLUSIONS}

In developing country like India, there is a lack of early and adequate antenatal care leading to high incidence of asphyxia. Moreover, majority of births occur at home without an assistance of trained birth attendants. The "Risk Approach" should be implemented with all seriousness to detect and offer special care to high risk mothers.

The above studied maternal and fetal factors which are strongly associated in causing asphyxia if detected early and managed appropriately can help in prevention of asphyxia related poor outcomes. Prematurity is significantly associated with birth asphyxia and also has higher incidence of asphyxia related mortality, hence all attempts should be made by treating obstetrician to prevent preterm births.

The prevalence of birth asphyxia represents only the "Tip of the iceberg." It has a wide range of impact on the further neurodevelopment of the child. This study thus concludes with the suggestion that we should be vigilant to the risk factors associated with perinatal asphyxia so that neonatal morbidity can be prevented with appropriate interventions including skilled resuscitation technique.

\section{BIBLIOGRAPHY}

1. American academy of pediatrics. Instructors manual for neonatal resuscitation. $5^{\text {th }}$ ed Elk Grove Village, IL and Dallas TX: American Academy of Pediatrics and American Heart Association;2006.

2. Ellis M, Costello A. Birth asphyxia, Apgar score and neonatal encephalopathy. Indian Pediatrics 1997 Nov;34:975-978.

3. Singh M. Diagnosis and management of perinatal asphyxia: current concepts. Indian Pediatrics 1994 Oct;31:1169-1174.

4. Lawn JE, Cousens S, Zupan J. 4 million neonatal deaths: When? Where? Why? Lancet 2005;365(9462):891-900.

5. Lawn J, Shibuya K, Stein C. No cry at birth: Global estimates of intrapartum stillbirths and intrapartumrelated neonatal deaths. Bull World Health Organ 2005;83:409-417.

6. Anne CC Lee, Mullany LC, Tielsch JM, et al. Risk factors for neonatal mortality due to birth asphyxia in southern Nepal: a prospective, community-based cohort study. Pediatrics 2008 Nov;121(5):e1381-e1390. 
7. Lahariya C, Paul VK. Burden, differentials and causes of child deaths in India.

Indian J Pediatr 2010;77:1312-1321.

8. Chandra S, Ramji S, Thirupuram S. Perinatal asphyxia: Multivariate analysis of risk factors in hospital births. Indian pediatrics 1997 Mar;34:206-212.

9. Daga AS, Daga SR, Patole SK. Risk assessment in birth asphyxia. J Trop Pediatr 1990;36:34-39.

10. Kaye D. Antenatal and intrapartum risk factors for birth asphyxia among emergency obstetric referrals in Mulago Hospital, Kampala, Uganda. East Afr Med J 2003 Mar;80(3):140-143.

11. Sasikala A, Raghavan S, Mishra N, Khatoon S, Bupathy A, Rani R. Perinatal outcome in relation to mode of delivery in meconium stained amniotic fluid. Indian J pediatr. 1995 Jan-Feb;62(1):63-67.
12. Nayak AH, Dalal AR. Meconium staining of amniotic fluidsignificance and outcome. Journal of Obstet and Gynecol 1990;480-483.

13. Oswyn G, Vince JD, Friesen H. Perinatal asphyxia at Port Moresby General Hospital: a study of incidence, risk factors and outcome.

PNG Med J 2000 Mar-Jun;43(1-2):110-120.

14. Thangwijitra S, Sripramodya C, Kanchanawat S. Risk factors of birth asphyxia in pregnancy 37 complete weeks and over by Apgar score less than 7 at 5 minutes. Thai J Obstet Gynaecol 2010 Apr;18(2):54-62.

15. Khreisat WH, Habahbeh Z. Risk factors of birth asphyxia: a study at Prince Ali Ben Al-Hussein Hospital, Jordan. Pak J Med Sci 2005 January-March;21:130-134. 